Reprinted from Hispanic Review, Vol. VII, No. 1, January, 1939

Printed in U. S. A.

\title{
LA INTRODUCCION DEL GRACIOSO EN EL TEATRO DE LOPE DE VEGA
}

$\mathrm{E}^{\mathrm{L}}$ L gracioso es uno de los personajes más importantes de la inmensa producción dramática de Lope de Vega. Trabajó en él más que en ningún otro personaje de toda su obra, pues es el que con más frecuencia se nos presenta. ${ }^{1}$ Empleó en su elaboración todo el talento de que le había dotado la naturaleza, y virtió en él los rasgos más sinceros de su carácter, pues si al gracioso se le quitan la bufonadas que exigía el vulgo, y se nos presenta en su verdadera intimidad, se verá que es el personaje más sincero y más humano de toda la obra de nuestro autor. Lope mismo nos atestigua su interés por el gracioso cuando al dedicar su comedia La francesilla a Montal-

${ }^{1}$ Computando los resultados de la investigación de la erudita alemana Maria Heseler, (Studien zur Figur des gracioso bei Lope de Vega und Vorgängern, Hildesheim, 1933) los cuales no acepto completamente, pero, que en general, nos pueden servir de guía, encontramos que de las 375 comedias de Lope que ella examina, 257 tienen graciosos. A pesar de la exagerada importancia que el gracioso asume en la comedia del siglo XVII, escasean los estudios sobre dicha figura. Véanse las obras siguientes a manera de bibliograffa: W. S. Hendrix, Some Native Comic Types in the Early Spanish Drama, Columbus, Ohio, 1925; J. P. W. Crawford, The Pastor and the Bobo in the Spanish Religious Drama of the XVI Century, Romanic Review, II, 1911; The Braggart Soldier and the Rufian in the Spanish Drama of the XVI Century, in ibid; S. E. Leavitt, Notes on the Gracioso as a dramatic Critic, Studies in Philology, IV, 1931; E. B. Place, Does Lope de Vega's Gracioso stem in part from Harlequin?, Hispania, 1934; J. F. Montesinos, Algunas observaciones sobre la figura del donaire en el teatro de Lope de Vega, en Homenaje a Menéndez Pidal, Madrid, 1925, II, 469-504; Ticknor, History, ed. 1849, II, 244 y sigs.; Schack, Geschichte, ed. 1854, 250 y sigs.; Klein, Geschichte des Dramas, Leipzig, 1871-75, X, 187; Wurzbach, Lope de Vega, und seine Komödien, Leipzig, 1899, 90; Martinenche, La comédia espagnole en France, Paris, 1900, 119120; R. Schevill, The Dramatic Art of Lope de Vega, Berkeley, 1918, 23-24; Pedro Muñoz Peña, El teatro del maestro Tirso de Molina, Valladolid, 1889, 29, 157, 208 Marjorie E. C. Bradford, The Gracioso of Lope de Vega, Radcliffe College, Ph.D. thesis, 1929-30, (inédita). 
bán dice: "repare de paso en que fué la primera en que se introdujo la figura del donaire, que desde entonces tanta ocasión dió a las presentes." ${ }_{2}$ Con razón dice Ticknor que debió haber producido un efecto notable, ${ }^{3}$ y con razón se vanagloria Lope de la introducción de dicho personaje que había de ser otra de las muchas riquezas que él había de legar a sus contemporáneos y a la posteridad dramática española.

Sin embargo, la crítica moderna ha puesto en tela de juicio el aserto de Lope, inclinándose a dudarlo. Castro dice: "Hasta que no se haga un fino estudio, cronológico y literario del gracioso en el teatro de Lope, no sabremos el valor (tal vez escaso) que deba concederse al aserto." 4 Federico Ruíz Morcuende: "Si reconocemos veracidad a la dudosa aserción hecha por Lope . . L La francesilla fué la primera comedia en que introdujo la figura del donaire." 5 Maria Heseler: "Die Angabe Lopes, dass er der Schöpfer des komischen Typus in Drama sei, ist ebensowenig wörtlich zu nehmen, wie seine andere über das erste Erscheinen des gracioso in der Francesilla. ${ }^{6}$ Vossler: "Den Dichter hat, wie so oft, auch hier sein Gedächtnis gestäuscht." Menciona a continuación algunos dramas que según él son anteriores a La francesilla, y concluye diciendo: "Abgesehen davon fällt es schwer zu glauben, dass Lope gerade in seiner Jugend, als er besonders stark unter dem Einfluss der italienischen Komödie und Stegreiftechnik stand und mit der Farcen der älteren Spanier schon sehr vertraut war, sich die Wirkung des Gracioso versagt haben sollte." 7 Finalmente, Buchanan se expresa igualmente con reservas: "The presence or absence of the gracioso may be an indication of the date of a play. . . . Some years ago, on internal evidence, I determined the date of this play (La francesilla) as 1598 . But there are plays written as early as 1594 , for example, El maestro de danzar, that contain the character. ${ }^{8}$

${ }^{2}$ Acad. N. V. 665 b.

${ }^{3}$ Ticknor, op. cit., 244. Hablando del interés que Lope tenía por el gracioso, dice Lord Holland, (Some Account of the Life, etc., London, 1806, 189): "Lope not only wrote but performed the part of such a buffoon at Valencia, in 1599 on the celebration of Philip III's nuptials." Holland se basa en la continuación de la Historia de Mariana, hecha por Miñana. La Barrera mantiene que esta afirmación no tiene ningún fundamento. Cf. Acad. I, 79. E. Juliá Martínez resuelve este enredo en Documentos, etc., en Bol. de la Real Acad., III, 1916, 540-560, en particular, 543, nota 4 .

${ }^{4}$ Rennert y Castro, Vida, etc., Madrid, 1919, 481 y 376 nota 1.

${ }^{5}$ Acad. N. X, Introd. vii.

${ }^{6}$ Heseler, Op. Cit., 14.

${ }^{7}$ Karl Vossler, Lope de Vega und sein Zeitalter, München, 1932, 302-3.

${ }^{8}$ M. A. Buchanan, Chronology, etc., Toronto, 1922, 6. 
Hay tantos errores cronológicos y de interpretación literaria en los argumentos que la crítica ha of recido para invalidar la afirmación lopesca, que se hace necesaria una revaluación crítica. Frente a estas dudas y negaciones nosotros volvemos a plantear el problema, enfocándolo desde un punto de vista diferente. ¿Es el Tristán de La francesilla el primer gracioso que salió de la pluma de Lope como él mismo dice? Para mantener este expostulado sería preciso definir al gracioso. Pero tal definición es imposible, debido a la complejidad de dicho personaje. ${ }^{9}$ Por lo tanto, analicemos los rasgos característicos de Tristán y aceptémoslo como fórmula o prototipo de graciosos, pues él fué el primero de su especie que Lope consideró digno de tal nombre.

\title{
Características de Tristán
}

1. Si no está borracho cuando aparece en escena, Tristán tiene fama de borrachón. Así pues, cuando empieza a disparatar, Feliciano dice: "Ya comienza este borracho." 10 Más tarde, para alentarlo, pues tienen que hacer un viaje por los Alpes, Feliciano le dice: "Tú los pasarás con vino," y Tristán se queja de que se bebe poco.

2. Tristán es cristiano, aunque no se nos presenta bebiendo y comiendo tocino para afirmar su cristiandad.

3. Parodia los amores y otros actos de su amo.

4. Parodia los discursos de Feliciano al igual que sus actos. largo discurso de despedida de Feliciano que termina:

\author{
Adiós, Arminda; adiós, celos, \\ papeles, gustos, amores, \\ pues sólo un taco de trucos \\ ha dado conmigo en Londres. ${ }^{11}$
}

${ }^{9}$ Dice Heseler en sus conclusiones (op. cit., 118): "Was wir am meisten an Lopes gracioso bewundern müssen, ist die ausserordentliche Mannigfaltigkeit." Place, (op. cit., 257) lo define asi: "To me the word gracioso denotes in its Golden Age theatrical sense a confidential servant who provides comic relief: (1) by making witty, humourously nonsensical, calculatedly stupid, or whimsically extravagant speeches, all of which are frequently ribald and which often have nothing to do with the plot; (2) by parodying his master's love affair; (3) by manifestations of hunger or gluttony; (4) by sometimes speaking a jargon or dialect; (5) by showing cowardice. In addition the gracioso gives advice to his master, sometimes to the extent of providing considerable plot motivation."

${ }^{10}$ Acad. N. V, 667 a.

${ }^{11}$ Acad. N. V, 570 a. No atinamos con el significado de este pasaje, por cuanto que Feliciano no va a Londres, sino a Francia. A menos que los versos en bastardillas no constituyan un idiotismo clásico, nos inclinamos a creer que Lope se identificó momentáneamente con Feliciano, recordando la expedición de la Invencible, que a decir verdad, tampoco llegó a Londres. De todos modos, Londres está más relacionado con Lope que con Feliciano. 
Adiós, amigos fingidos, moneda que ahora corre, y si verdadero alguno, mi destierro sienta y llore . . .

sigue la parodia de Tristán:

Adiós, tabernas de corte, galera en que yo solía fundar mis estanteroles; lavaderos y pilares, baratillo y herradores. Adiós, Juana, que sin duda me has pegado lamparones, pues voy a Francia a curarme en un rocín matalote. ${ }^{12}$

5. El es presuntuoso. Se precia de saber mucho, cuando, en realidad no sabe nada. Le gustan los términos altisonantes que, como es natural, pronuncia erróneamente. ${ }^{13}$

6. Tristán es el encargado de recitar los pasajes picantes de la pieza. ${ }^{14}$ El resultado es que a veces su lenguaje es muy bajo.

7. Tristán se las echa de políglota. ${ }^{15}$

8. A veces se esfuerza por ser gracioso. Entonces lo vemos empleando retruécanos, forjando imágenes extravagantes, fingiendo no entender lo que se le dice, etc. Por ejemplo, Feliciano se acuerda de Madrid y exclama:-¡Ah Prado mío!-y Tristán responde:

Bien haces, como jumento te acuerdas del lugar adonde paces. ${ }^{16}$

9. Este ejemplo demuestra que a veces él es también irrespetuoso para con su amo. ${ }^{17}$

10. Es refunfuñón. En sus apartes graciosos, dichos al público, él se burla de su amo y llega así a simpatizar con el auditorio. Así vemos que después que Alberto, padre de Feliciano, ha dictado la sentencia que lo destierra de su patria, Tristán se burla de su amo a guisa de consolación:

${ }^{12}$ Acad. N. V, 670 a.

${ }^{13}$ Ibid. 667 a.

14 Ibid. 698 a.

${ }^{15}$ Ibid. 695 a.

${ }^{16}$ Ibid. 675 a.

${ }^{17}$ Ibid. 678 a. 
Feliciano. ¿Qué estás hablando entre dientes?

Tristán. ¡Sucesos, por Dios, notables!

Feliciano. ¿De qué te ríes?

Tristán.

De verte

con mil escudos en oro. ${ }^{18}$

11. El, no solamente se burla de su amo en su misma cara, sino que se burla de él a sus espaldas:

le dió cierta señora vino estítico,

Desde el miércoles

y, como amante enfermo del estómago,

anda el pobrete con aquellos báguidos,

mirando al cielo que parece astrólogo.19

12. Tan acostumbrado está a burlarse de la gente, que si no halla de quien burlarse, se mofa de sí mismo:

Y a mí con jamón que sea purísimo,

que me tiene Amor acabadísimo. ${ }^{20}$

El se burla de su propio estado y linaje:

Soy camarada y amigo

de este hidalgo, criado digo,

que siempre adelante paso;

digo, que él viene tras mí,

él a caballo y yo a pie. ${ }^{21}$

Dorista. ¿Es caballero?

Tristán.

$$
\text { ¿Yo? No. }{ }^{22}
$$

13. Tristán siente un desvelo constante por el dinero. Bastante que le romperá la cabeza a Feliciano por haber perdido sus escudos. Y aunque éste lo consuele, nada lo convencerá:

¡Ojos que los vieron ir,

No los verán más en Francia. ${ }^{23}$

${ }^{18}$ Ibid. 669 a.

${ }^{19}$ Ibid. 678.

${ }^{20}$ Ibid.

${ }^{21}$ Ibid. 676.

${ }^{22}$ Ibid.

${ }^{23}$ Ibid. 676 b. El gracioso representa ciertas tradiciones populares que se manifiestan en la citación de refranes y viejos romances como éste. Estos versos que anotamos, procedentes de uno de los romances a Belerma, (Romancero General, $B A E, I, 260)$ se hicieron proverbiales, como lo prueban las siguientes citas: Lope, El anzuelo de Fenisa, H. III, 384 c; La serrana de la Vera, Acad. XII, 8-9; Tirso, Palabras y Plumas, BAE V, 3 a; Quiñones de Benavente, NBAE, XVIII, 814 a; Castillo Solorzano, Tardes entretenidas, (Col. de Nov. Antiguas Esp.) ed. Cotarelo, p. 181. 
14. Si Tristán "sueña con los cuartos," su eterna pesadilla es la comida. Tan pronto ve el dinero, lo primero que se le ocurre es:

Métete en cas de un figón

y comamos como grandes. ${ }^{24}$

15. El es indiscreto. Fué debido a su indiscreción que las damas francesas les pescaron los cuartos. ${ }^{25}$

16. Sin embargo, él es astuto y saca a Feliciano de los aprietos en que se mete.

17. Las intromisiones de Tristán son características de todos los gracioso. Este resorte cómico es muy común, pues da lugar a la desesperación del galán. Este contraste entre el galán desesperado y colérico y el criado entrometido se encuentra en muchas ocasiones en La francesilla.

18. El es incrédulo. No cree en pamplinas mitológicas, ni hasta en la historia misma. Cuando Feliciano recurre a la antigüedad y a la mitología para justificar la burla que Clavela y Dorista les hicieran, Tristán responde:

$\begin{array}{ll} & \text { ¿Qué diablos me están diciendo } \\ & \text { inútiles bernardinas } \\ & \text { en tiempo que a pie caminas } \\ & \text { y de hambre vas muriendo? } \\ & \text { ¡Ah, vieja de Satanás, } \\ & \text { pescador con pie de cabra! . . } \\ \text { FELICIANo. } & \text { Así hicieron las arpías } \\ \text { Tristán. } & \text { los manjares de Fineo. } \\ & \text { ¡Historias! . . . }{ }^{26}\end{array}$

19. No solamente las necesidades físicas ocupan los pensamientos de Tristán, sino que se desvela también por la comodidad física en general.

Feliciano. ¿No hizo César romano más indignos desatinos?

Tristán. No iba a pie por los caminos, las espuelas en la mano...$^{27}$

20. La belleza de Clavela deslumbra a Feliciano, pero no así a Tristán. El clasifica a todas las mujeres en la misma categoría: volubles, indignas de causar el desvelo de los hombres.

${ }^{24}$ Acad. N. V, 669 a y b.

${ }^{25}$ Ibid. 676 a.

${ }^{26} \mathrm{Ibid} .677 \mathrm{a}$

${ }^{27}$ Ibid. 
Feliciano. Esta no es mujer, es sol.

Tristán. Sea de invierno, porque pasa

presto aunque tarde amanece. ${ }^{28}$

El no excluye a ninguna. Véase de qué figura se vale para expresar su opinión de Arminda:

El caldero sube y baja;

pero el pozo no se mueve.

Ansí será Madrid y ella:

él quedo y ella mudable. ${ }^{29}$

21. Tristán es el consejero de su amo, especialmente en cuestiones de amor. Dada la opinión que él tiene de todas las mujeres en general, se comprenderá fácilmente que su consejo será contra el amor, o en pro del fácil amor. Cuando se encuentran en un aprieto a causa de la aventura con Clavela, Tristán le dice a Feliciano:
Bueno fuera haber callado
y no tener que llorar, que el ganso por el graznar
muere a manos del soldado. ${ }^{30}$

Ya él le había aconsejado anteriormente que no se metiera en asuntos de amor, que nunca tienen buenos resultados.

22. Como se ve del ejemplo anterior y como ya dejamos apuntado (nota 23), Tristán es amigo de refranes, moralejas, etc. ${ }^{31}$

23. Tristán, como todo buen gracioso, es embustero; pero sus mentiras son inofensivas.

24. El es cobarde por naturaleza. En cualquier peligro en que se encuentra, él se acuerda de que es mortal. Así lo vemos dándole consejos a Feliciano: "Si no huyes, ten por cierto que no escapas de ser muerto." ${ }^{32}$ Este consejo lo da él pensando en sí mismo.

25. Tristán, como todo buen gracioso, es leal criado. Pero no hay que llevar esta idea a la exageración. El seguirá a su amo a prueba de hambres y malos tratos, como Montesinos nota, pero su lealtad reconoce límites. En esto, Lope sigue a Torres Naharro, cuyo gracioso, Boreas, abandona a Himeneo cuando ve que el Marqués sale, espada en mano, furioso, a vengar la deshonra de su hermana. Cuando la cobardía del criado se encuentra en conflicto con su lealtad, por lo general, la última cede. El instinto natural

\footnotetext{
${ }^{28}$ Ibid. 675 a.

${ }^{29}$ Ibid. 674 b. Cfr. 692 a.

${ }^{30}$ Ibid. 685 b.

${ }^{31}$ Ibid. 685 b, 699 b.

${ }^{32}$ Ibid. 684 b.
} 
prevalece en él como humano, y muy humano, que es. Cierto, que él está siempre animado de los más nobles propósitos. Así vemos a Tristán exclamar: "Dame señor, muerte a mí y deja a Pedro, señor!" Pero esos arranques no son más que payasadas. Tan pronto como él ve que Alberto hace el menor movimiento, exclama: "¡Ay, muerto soy!"

26. Esos arranques de valor no son, en el gracioso, ni más ni menos que farfantonerías. El se las quiere echar de bravo; por eso intercede. La bravuconería es una de la características distintivas del gracioso. Así vemos a Tristán haciéndose el ofendido:

\author{
Buena está la borrachera, \\ Perote con mi fregona... .$$
\text { ... y ella, mona, }
$$$$
\text { anda buena virotera. }
$$$$
\text { ¡Entrese allá enhoramala } \text { !3 }^{33}
$$

El lector que sabe que Pedro y Perote no son más que Clavela, disfrazada de hombre, comprende realmente las sandeces de Tristán. A esta actitud nada mejor que la pregunta de Clavela: "¿La paz de Francia te enoja?"

27. El campea libremente por toda la pieza.

28. Está estrechamente ligado, con los personajes principales de la comedia, a la traza, tanto, que en muchos casos él es quien resuelve los enredos que se presentan en el desarrollo de la trama, eg: él es quien descubre la identidad de Clavela, que está disfrazada.

29. Si Lope fuera a seguir la técnica de Torres Naharro, Tristán terminaría el drama; pero no es así. Sin embargo, él es quien recita los últimos versos de los dos primeros actos, en los cuales se menciona el título de la comedia: "¡Qué graciosa francesilla!"

30. Finalmente, junto con el casamiento del galán y la dama se procede al inevitable matrimonio del lacayo y la sirvienta, aunque en esta comedia la sirvienta no es Dorista, criada de Clavela, sino Juana, sirvienta de la casa de Feliciano.

Estas son, en resumen, las características sobresalientes del gracioso de La francesilla. Sobre el sentido filosófico del gracioso, Montesino ha dicho lo siguiente, que es en todo aplicable a Tristán: “... el gracioso y el galán son como dos partes diametrales que pugnan dolorosamente por aprehender la realidad. El lacayo enseña a su amo a olvidar; pero el espíritu del afligido escapa aquel bienaventurado esfuerzo para dejarse atormentar voluptuosamente por sus penas de amor. El uno no para mientes sino en las miserias;

${ }^{3}$ Ibid. 698 a. 
el otro no tiene alma sino para las excelencias. Carne y espíritu. No es posible entenderse. Lo impide la contextura espiritual de ambos. Es la nobleza o la plebeyez, la sangre." ${ }^{34}$

Dramas de Lope anteriores a La francesilla.

La francesilla fué escrita en 1595 y no en 1598, como hasta hace poco se creía $^{35}$ Partiendo de esta fecha examinemos ahora, a la luz del análisis que de Tristán hemos hecho, los personajes cómicos que lo preceden que los críticos han aceptado como graciosos.

Veintiseis comedias hay atribuidas a Lope que se suponen o que se sabe fueron escritas antes o durante el año 1595. Estas son:

1574? El verdadero amante

1579?-83? Hechos de Garcilaso y el moro Tarfe

La Pastoral de Jacinto (¿primer drama en tres actos?)

1587? Belardo furioso

1587? El soldado amante

1587-98 El cerco de Santa Fe

1588-95 El hijo venturoso

1588-95 La infanta desesperada

1588-95 El ganso de oro

1588-95 El nacimiento de Ursón y Valentín

1590? El grao de Valencia

1593? Los enredos de Benito

${ }^{34}$ Montesinos, Op. Cit., 489. Al decir "carne y espíritu" lo primero que se nos viene a la mente son los sinónimos respectivos, Sancho y Quijote. En el desarrollo de su estudio el Sr. Montesinos los aludió inevitablemente. Bien pudo añadir que Lope no pasó por alto este parecido. El desprecio agradecido, H. II, 253.

Inés Pues un libro y esta vela os será de gran provecho.

D. Bernardo. ¿Quién es?

Inés Parte veintiseis de Lope.

D. Bernardo. Libros supuestos que con su nombre se escriben.

Sancho. Y a mí, por si no duermo, ¿qué me dais?

Inés. A Don Quijote, porque vos y vuestro amo imitáis sus aventuras.

Montesinos cita otro pasaje de La cortesía de España, Acad. N. IV, 352 b, sin hacer hincapié en este punto. 73-76.

${ }^{25}$ Cfr. J. H. Arjona, La fecha de La francesilla, en Hispanic Review, V, 1937, 
1594? La venganza venturosa

1594 San Segundo de Avila

$1594 \quad$ Laura perseguida

ca. 1595 Jorge toledano

1590-95 Los amores de Albania e Ismenia

ca. 1583 La defensa en la verdad

1587? La ingratitud vengada

1588-89 Más vale salto de mata que ruego de buenos

1590-95 El dómine Lucas

a 1593 Los comendadores de Córdoba

$1593 \quad$ La tragedia del rey $D$. Sebastián y bautismo del principe de Marruecos

1593 El favor agradecido

1594 El leal criado

1594 El maestro de danzar

Las primeras diez y siete comedias de esta lista no ofrecen particularidad alguna que justifique un análisis detallado del elemento cómico. En casi todas ellas sentimos la vivaz musa de Lope hilvanando escenas cómicas, creando personajes más o menos risibles, inventando chistes y retruécanos, difundiendo, en general, el elemento del donaire que tan importante papel había de desempeñar poco después al concentrarse en el personaje del gracioso. En $E l$ grao de Valencia hallamos un pícaro jocoso. En El ganso de oro encontramos dos rufianes: Matracón y Tragafieros, un alcahuete, un soldado viejo, cojo, manco y tuerto, un gitano y dos rameras. En La infanta desesperada hay dos hombres y una mujer presos. En Belardo furioso el elemento del donaire es mucho más extenso. Belardo marcha por toda la pieza, haciendo y deshaciendo en sus locos devaneos que contagian a los demás personajes de la comedia; pero éstos no son, ni en los unos ni en el otro, esencialmente graciosos. Sin embargo, Bato, personaje de mínima importancia, que el poeta apenas bosqueja, sugiere algunos de los rasgos característicos de la figura del donaire. Alguno que otro elemento cómico que hay en la pieza se halla disperso por todas partes. Todos los personajes comentan alguna gracia. Pero falta en la comedia la centralización del elemento cómico en un personaje solo que podamos llamar el gracioso. De Laura perseguida dice Place, "There is no gracioso as such, but merely a comic rustic of bobo order who figures in two scenes." 36 Dúdase en extremo que Las burlas y enredos de Benito sea de Lope. Dice Américo Castro: "La atribución a Lope requiere

${ }^{36}$ Place, op. cit., 261. 
detenido estudio." 37 Cotarelo: "Del examen interno de esta comedia se deduce que no debe pertenecer a Lope, sino más bien a un poeta andaluz." ${ }^{38}$ Además, y esto es lo que nos importa, como ya Rennert y Castro han notado, no tiene la comedia figura del donaire. $^{39}$ En El hijo venturoso, Leonardo, loco de amor comete insensateces que un sentido de humor pervertido llamaría graciosas. Hämel no le dedica ni una palabra al elemento cómico de esta comedia en su estudio. ${ }^{40}$ En fín, no creemos necesario pasar revista de todas las comedias de la primera parte de nuestra lista pues el elemento del donaire es escaso y si lo hay, Lope no lo depositó expreso en ningún personaje para llamarlo el gracioso de la comedia. ${ }^{41}$

Las comedias del segundo grupo merecen discusión aparte, pues son éstas las que según la crítica ofrecen ejemplos de gracioso anteriores al Tristán de La francesilla.

\section{LA DEFENSA EN LA VERDAD}

Heseler ${ }^{42}$ y Vossler ${ }^{43}$ le atribuyen un gracioso a esta comedia. Tanto uno como la otra se conforman con referirla al año de 1583, o poco después, a causa de la relación de la batalla naval de las Islas Terceras que se hace en el primer acto. Tanto uno como la otra desconocen o ignoran por completo las reservas de Cotarelo sobre la paternidad lopesca de esta comedia. ${ }^{44}$ Y lo que es más importante, ninguno de los dos críticos alemanes ha notado las peculiaridades de la versificación que hace la fecha de 1583 absolutamente imposible. He aquí el análisis total de la versificación: romance, $59 \%$, redondillas $24 \%$, pareados $15 \%$. Sin hacer hincapié en que la parquedad de formas poéticas no delata la versátil pluma de Lope, es evidente que el exagerado porcentaje de romance indica una fecha mucho más tardía de la que los críticos alemanes le atribuyen a esta comedia. El análisis de la versificación indica que esta comedia

${ }^{37}$ Rennert y Castro, op. cit., 466.

${ }^{38}$ Acad. N. IV, Introd. viii.

${ }^{39}$ Rennert y Castro, op. cit., 466.

${ }^{40} \mathrm{~A}$. Hämel, op. cit.

${ }^{41}$ C. Bruerton, Lope's Belardo-Lucinda Plays en Hispanic Review, V, No. 4, 1937, enumera sólo veintiún dramas auténticos escritos antes de 1599. Nuestra lista se dilata mucho más porque hemos incluído dramas auténticos y dudosos y algunos cuyas fechas no han sido establecidas definitivamente. Pero si se hallara más tarde que algunas de estas comedias son posteriores a 1595, esto no perjudica nuestra exposición, pues Lope excluyó el gracioso de muchas comedias posteriores a La francesilla.

${ }^{42} \mathrm{Op}$. cit. 14.

${ }^{43}$ Op. cit. $302-303$.

"Acad. N. IV, Introd. xvi. 
debió haber sido escrita o refundida entre el 1620 y 1630.45 Por lo tanto no es necesario averiguar si Trigueros es o no es un gracioso completo, lo cual, dicho sea de paso, es dudabilísimo.

\section{La tragedia del Rey don Sebastián y bautismo del PRINCIPE DE MARRUECOS}

Heseler ${ }^{46}$ incluye esta comedia dos veces en la lista final de su Studien. La incluye primero entre los dramas de la Parte XI con el título de El principe de Marruecos; le atribuye la fecha de 16021603, que copia de Buchanan, y dice que no tiene gracioso. La vuelve a incluir entre las comedias sueltas, con el título de La tragedia del Rey Don Sebastián; le atribuye la fecha de 1593, que copia de Menéndez y Pelayo, ${ }^{47}$ y le asigna un gracioso, Alonso, villano. ¡Vamos, hombre! El tal Alonso no es gracioso, ni cosa que se parezca. El es un campesino timorato que figura en sólo una escena, donde unos ladrones le roban el burro en que cabalga levantándole en vilo sobre la albarda sin que él se dé cuenta. Ya Rennert notó: "A few jests are assigned to Zulema and to the villano Alfonso (sic), but there is no true figura del donaire in the play."

\section{Más vale salto de mata que ruego de buenos}

$\mathrm{Al}$ editar esta comedia, Cotarelo dice: "El principio de un romance que canta Mendoza, criado de don Carlos, 'Hortelano era Belardo, en las huertas de Valencia,' nos pudiera indicar que esta comedia, fruto de la mocedad de Lope, fuese escrita en Valencia entre 1588 y 1590." 48 Vossler ${ }^{49}$ y Heseler ${ }^{50}$ desconocen o ignoran nuevamente la fuerza del subjuntivo y mantienen que esta comedia fué escrita para la fecha en que Cotarelo dice que pudo haber sido escrita; que Mendoza es un gracioso, y que por lo tanto, Lope se equivocó en su dedicatoria de La francesilla.

La cita del romance prueba solamente que la comedia fué escrita después de la fecha mencionada pero no establece en modo alguno el

${ }^{45} \mathrm{El}$ Sr. S. G. Morley me sugiere amablemente, anticipando resultados de la extensa cronología de Lope que prepara con el Sr. C. Bruerton, que esta comedia debió haber sido escrita después de 1625 , y que además el texto actual no parece ser de Lope.

${ }^{46}$ Op. cit. 126 y 132.

${ }^{47}$ Sobre la fecha de esta comedia, Cfr. J. H. Arjona, Dos errores de cronología lopesca, en Romanic Review, Vol. XXVII, No. IV, 1937.

${ }^{48}$ Acad. $N$. VII, Introd. xx.

${ }^{49} \mathrm{Op}$. cit. 302.

${ }^{50} \mathrm{Op}$. cit. 14. 
terminus ad quem de la composición de la comedia. Esta misma cita aparece en Las paces de los reyes, 1604-1617 y en Al pasar del arroyo, $1616 .^{51}$ Además, el análisis de la versificación prueba concluyentemente que esta comedia no pudo haber sido escrita en época tan temprana: redondillas $41 \%$, romance $36 \%$, décimas $3 \%$, liras $5 \%$, sueltos $4 \%$, y quintillas $8 \%$. El excesivo porcentaje de romance, la presencia de décimas ${ }^{52}$ y la escasez de quintillas son indicaciones patentes que Más vale salto de mata . . . no pudo haber sido escrita sino en la segunda década del siglo diez y siete. ${ }^{53}$ Nótese además otra característica notable: los tres actos terminan en pie de romance.

Descartadas pues, estas tres comedias, que por errores cronológicos la crítica había introducido en este período de la vida de Lope, concretémonos a discutir las últimas cinco comedias de nuestra lista, ${ }^{54}$ cuyas figuras cómicas son los verdaderos precursores del gracioso. Este se incuba en la mente lopesca en los años 1593-1594.

${ }^{51}$ Cfr. C. Bruerton, On the Chronology of Some Plays by Lope de Vega, Hispanic Review, III, No. 3, 248. La fecha de Al pasar del arroyo, que Bruerton cita como 1615-1616, ha sido establecida finalmente por W. L. Fichter, en Hispanic Review, (mismo número) como el 29 de enero de 1616.

${ }^{62}$ Cfr. Juan Millé y Giménez, Sobre la fecha de la invención de la décima o espinela, en Hispanic Review, V, No. 1, 1937.

${ }^{53}$ Aquí el Sr. S. G. Morley me sugiere una vez más que esta comedia debí haber sido escrita entre 1612 y 1615 y que el texto presente no parece ser de Lope.

${ }^{4}$ Por mantener el método que nos hemos propuesto discutiremos sólo en forma de nota el caso de La ingratitud vengada.

Ni Rennert ni Castro le atribuyen gracioso a esta comedia. Heseler incluye a Rodrigo, lacayo de esta comedia, entre los graciosos de Lope. (Op. Cit. 127) Place, (Op. Cit. 260) niega esta atribucion: "Of Lope's three most primitive extant comedias (El verdadero amante, La pastoral de Jacinto and Los hechos de Garcilaso de la Vega y moro Tarfe), all written before 1585 , none has a gracioso even in embryo. The same may be said of La ingratitud vengada and Belardo furioso (both possibly of 1587) . . . La ingratitud vengada does have four rufianes and a lackey named Rodrigo who comes on stage dancing in one scene, but this lackey is in no sense a gracioso as defined above."

El elemento del donaire de esta comedia se halla esparcido por todas partes. Varios personajes hay en ella en que se encuentran esbozados algunos de los rasgos característicos del gracioso. Estos personajes son los rufianes mencionados: Garrancho, Cespedosa, Barboso, y Matamoros, los cuatro, fanfarrones de la ralea que se encuentra en Rinconete y Cortadillo. Quizás si fuera éste el aspecto de la comedia que atrajo a Cervantes en su crítica que de ella hace en el Quijote.

Alejo y Belardo son dos pajes que nos hacen sonreir con sus nimiedades. Corcina, es una madre alcahueta, que nos recuerda'a la Tía fingida. Finalmente, Tancredo y Rodrigo son dos criados que según algunos críticos se disputan el honor de ser la figura del donaire. Rodrigo tiene menos de gracioso que Tancredo, y aún éste no puede llamarse un gracioso. En él encontramos el intento de la parodia de las acciones del galán. Sin embargo, la parodia es tan convincente, 


\section{El dómine Lucas}

Decio, capigorrón de esta comedia, no es un gracioso completo, pero su desarrollo indica que no es uno de los primeros intentos de Lope. Basándonos en el desarrollo de este personaje, concordamos con Castro en que la fecha de esta comedia debe de ser $1593 .{ }^{55}$

Decio aparece por primera vez "con una sotanilla muy raída, y otra hecha pedazos debajo, y un mal sombrero y un mal ferreruelo," a medianoche, en el centro de una plaza desierta, haciendo un recado:

¡Qué hora para enviar un forastero a la plaza! ${ }^{56}$

Sale muerto de miedo, pero animándose a sí mismo, se las echa de bravo. Cuando Floriano, su amo, le sale al encuentro, para robarle la sotana, pues se quiere disfrazar, entonces, ¡Aquí fué Troya! Decio habla hasta latín: "Logicam audio et sum ego compostellanus!" ${ }^{57}$ dice él, queriéndose hacer pasar por estudiante salmantino. - ¿Gallego?-pregunta Floriano.-Maxime-responde Decio, y así, por el estilo, continúa la conversación en latín chapurrado. Toda esta escena está llena de un humorismo muy sabroso.

Decio podrá ser todo lo fiel que se quiera; pero con tal de salir bien de un aprieto, él no respeta ni a su amo. Así lo vemos insultando a Floriano disfrazado:

$$
\begin{gathered}
\text { Eso es llano, } \\
\text { sino que siendo villano, } \\
\text { presume venir del Cid. }{ }^{58}
\end{gathered}
$$

Floriano se enfada, le pide que suelte la capa, si no quiere volver al mesón con la cabeza rota. Y naturalmente que Decio arroja la sotana y todas las vestiduras que tenía y se va refunfuñando: "Nudus salí del mesón, ed illus revertar nudus." ${ }_{59}$

En el segundo acto, ya pasada toda la tempestad, Decio se nos presenta como una figura simpática. Encuentra a su amo en un estado lamentable y al reconocerlo por la dichosa sotana, le pregunta:

que se convierte en realidad, y el criado, no el galán, es el que termina por merecer la mano de la protagonista. Esto es indudablemente algo muy ajeno a la psicología del gracioso que si a veces le haçe la corte a la dama principal, es solamente a manera de mofa.

${ }^{65}$ Rennert y Castro, op. cit., 34, nota 1 .

${ }^{56} \mathrm{H}$. I, 44 a.

${ }^{57}$ Ibid. 46 a.

${ }^{68}$ Ibid.

${ }^{59}$ Ibid. $46 \mathrm{c}$. 
“No reconoces, señor, a tu antiguo servidor?" Hacen las paces, y Floriano se alegra de que haya llegado tan a buen tiempo para llevar una carta a Salamanca.

$\begin{array}{ll}\text { Decio. } & \text { No estoy para caminar. } \\ \text { Floriano. } & \text { ¿Cómo? } \\ \text { Decio. } & \quad \text { Estoy sin blancas. } \\ \text { Floriano. } & \text { No te faltará dinero. } \\ \text { Decio. } & \text { Y sin él te he de servir. }{ }^{60}\end{array}$

He aquí, una vez más, la lealtad del lacayo puesta de relieve. El hará cualquier cosa por su amo. Sus sentimientos son, por lo general, nobles, si son espontáneos; pero una vez que se da de frente con la realidad, sus sentimientos desaparecen momentáneamente para reaparecer pasado el peligro, como las aguas de un río que se sumergen al pie de una montaña para volver a brotar bien pronto más frescas y claras.

Ultimamente encontramos a Decio en un mesón, tratando de arreglar asuntos de su amo. Comienza alzando la voz, pero ve que el tabernero le contesta con altanería "¡Por el Duque don Bribón, que te deshaga la faz!" El se amansa y propone: "Averigüémoslo en paz." ${ }^{61}$ Encontrándose con el obstáculo, él cede, y pensando en el bien de su amo, él revela su secreto, añadiendo: "Aunque sé que de matarme, sin deber por eso pena." ${ }^{2}$ Tan pronto como aparece Floriano, las cosas cambian de aspecto. Ahora no es el mesonero el que lo va a intimidar con gritos. Decio sabe que su amo lo defenderá y ahora se da aires de importancia.

En fin, terminaremos esta discusión de El dómine Lucas asegurando que Decio no es un gracioso completo. Cierto que él reúne algunas de las características de la figura del donaire, pero éstas son tan pocas que no le conquistan el título de gracioso. El aparece solamente en la sexta escena del primer acto, en la décimacuarta del segundo, y en las décimaquinta y décimaoctava del último. Está ligado en parte a la trama de la obra, pero no marcha al compás de su amo parodiando sus actos o sus diálogos. Ni siquiera está presente al final de la pieza.

Decio ha atraído la atención de algunos críticos. Rennert cita a Chorley que duda si el personaje de Decio puede ser llamado gracioso o no. ${ }^{63}$ Buchanan y Castro pasan la cuestión por alto. Heseler lo incluye en su lista de graciosos, ${ }^{64} \mathrm{y}$, finalmente, Place

${ }^{60} \mathrm{Ibid} .46 \mathrm{~b}$.

${ }^{61}$ Ibid. 64 a.

${ }^{62}$ Ibid.

${ }^{63}$ Rennert, op. cit., 504.

${ }^{64}$ Heseler, op. cit., 127. 
niega que Decio sea un gracioso, ${ }^{65} \mathrm{Y}$ tiene razón pues Decio es solamente un precursor de la figura del donaire.

\section{El leal CRIAdo}

Heseler incluye a Julio en su lista de graciosos. ${ }^{66}$ Vossler no está seguro: "Im Leal criado den Lope am 24 Juni 1594 in Alba de Tormes abgeschlossen hat, kommt ein dienender Begleiter des Helden namens Julio vor, dem mit seinen Wortwitzen und Parodien der Haupthandlung so gut wie nichts zum richtigen Gracioso fehlt." ${ }_{67}$ Rennert: "This play has no true figura del donayre. The criado leal of the title is Uberto, and not the servant or criado Julio who is also one of the personages. Julio has some few witticisms alloted to him, but the gracias are soon abandoned." 68 Place: "El leal criado exhibits a servant, Julio, who makes comic speeches and parodies the main love action but is not the Loyal Servant of the title rôle and in general is a very secondary character." 69

Esta actitud titubeante de la crítica se debe a que Lope juguetea con la idea del gracioso sin decidirse completamente. En Julio se reúnen contadas cualidades del gracioso, pero le faltan muchas de las marcas distintivas de dicho personaje. Su presencia es escasísima en la comedia. El apenas si sigue a su amo. En segundo lugar, la clásica pareja de la dama y el galán está relegada a segundo término de importancia, pues el verdadero interés del drama es subyugar todas las acciones a los actos de lealtad de Uberto, modelo de criados leales. El drama que es de por sí de pobre contextura, relega a Julio a lugar de mínima importancia, y por lo tanto, le resta la cualidad esencial del gracioso: su importancia en la acción. Por consiguiente, honramos a Julio lo suficiente con ponerlo junto con Decio entre los precursores del gracioso.

\section{El MAESTRO DE DANZAR}

Este es uno de los dramas que más ha dado que hacer a los críticos. Buchanan, ${ }^{70}$ Heseler, ${ }^{71}$ y Federico Ruiz Morcuende ${ }^{72}$ afirman

${ }^{65}$ Place, op. cit., 261. "Of the four plays written between 1588 and 1595 ( $E l$ domine Lucas, etc. . . . ) none has a gracioso . . . El dómina Lucas exhibits in two scenes a student flunky or capigorrón whose clothes are stolen by the chief character in order to pose as a student tutor."

${ }^{66} \mathrm{Op}$. cit. 127.

${ }^{67}$ Op. cit. 302.

${ }^{68}$ Op. cit. 514.

${ }^{69}$ Op. cit. 261.

${ }^{70}$ Chronology, 6.

${ }^{71}$ Op. cit. 131 .

${ }^{72}$ Acad. N. X, Introd. vii. 
que El maestro de danzar tiene un gracioso. Rennert dice sencillamente: "The servant Belardo suggests the gracioso." ${ }^{73}$ Place lo analiza de este modo: "El maestro de danzar has likewise a very minor comic character, Cornejo, who seems to have scant connection with the plot." Dice Lope en sus acotaciones: "Salen Aldemaro, Belardo y Cornejo, escudero armado a lo gracioso." Place cree que "these are the earliest examples . . . in Lope's plays of the use of the word gracioso tending toward its ultimate connotation." 74 Castro: "En El maestro de danzar, compuesta a más tardar en 1594, el criado Belardo (Cornejo en la fe de erratas, pág. 543) representa el tipo del gracioso." 75 En la misma página, entrada diferente, "En El maestro de danzar, el escudero Cornejo se parece mucho a un gracioso."

Pues bien, el Cornejo de esta comedia no es más gracioso que ninguno de los que venimos examinando. El es cobarde, burlón, comilón, amigo de la exageración y los retruécanos, y se las echa de sabihondo, pero todas estas características están muy ligeramente insinuadas. Parecen rasgos de un pincel tímido, no seguro de sí mismo, que titubea antes de esparcir la pintura en el lienzo. Cornejo es un personaje demasiado insignificante en la pieza. Su papel es muy corto y no contribuye en nada al desenvolvimiento o desenlace de la acción. Por eso, calificarlo de gracioso equivaldría a dilatar exageradamente el sentido de la palabra. Rennert lo describe acertadamente cuando dice que Cornejo augura el tipo del gracioso.

\section{Los COMENDADORES de C6́Rdoba}

Sabemos que Gabriel Núñez se comprometió el 9 de julio de 1593 a representar una comedia titulada Los comendadores. Con este título la incluye Lope en su primera lista del Peregrino. Sin embargo, no podemos afirmar categóricamente que Los comendadores y Los comendadores de Córdoba sean una misma comedia, o hasta qué punto sea el texto que poseemos copia fiel del original lopesco. ${ }^{76}$

Por equivocación Castro mantiene que esta comedia no tiene figura del donaire. ${ }^{77}$ El Galindo de esta comedia es tan gracioso como Tristán o cualquier otro gracioso consumado de Lope. Una

${ }^{73} \mathrm{Op}$. cit., 508.

${ }^{74}$ Op. cit., 261.

${ }^{75}$ Op. cit., 481.

${ }^{76}$ Aquí tambén los Sres. Morley y Bruerton me sugieren que aunque es posible que Los comendadores citada en El peregrino sea la misma de Los comendadores de Córdoba, esta comedia difiere de la citada por Núñez pues el análisis de la versificacón indica que fué escrita en fecha posterior a 1593.

${ }^{77}$ Ibid. 470 . 
somera lectura de la comedia basta para convencernos. Cierto es que Galindo tiene un fin trágico, muriendo a manos del Veinticuatro que rabiando de celos mata a cuanto bicho viviente alberga su casa. Pero no hay que extrañarse, pues ya Montesinos ha mostrado que muchos graciosos mueren con el donaire en los labios. ${ }^{78}$

Sin embargo, Los comendadores de Córdoba representan un caso excepcional en la carrera literaria de Lope. El poeta que se vanagloria de escribir tantas comedias, que dispersas por el mundo no vuelven nunca a él, o si vuelven llegan tan estragadas que él no las reconoce, parece haber tenido afecto especial por esta comedia toda su vida. Prueba de ello es que si la escribió en 1593 o antes, la debió haber tenido presente al editar sus Rimas en 1602, pues en ellas incluye dos de sus sonetos. ${ }^{79}$ La recordó en 1603 al incluirla en la primera lista del Peregrino. La recordó en 1609, cuando fué publicada. Millé y Giménez cree que el texto actual data de esta fecha. La introducción de décimas, que no aparecen, según él, hasta 1599, justifica su conjetura. ${ }^{80}$ Finalmente, Lope le dedicó otro recuerdo a esta comedia en El cuerdo en su casa, que se supone escrita después de $1598 .^{81}$

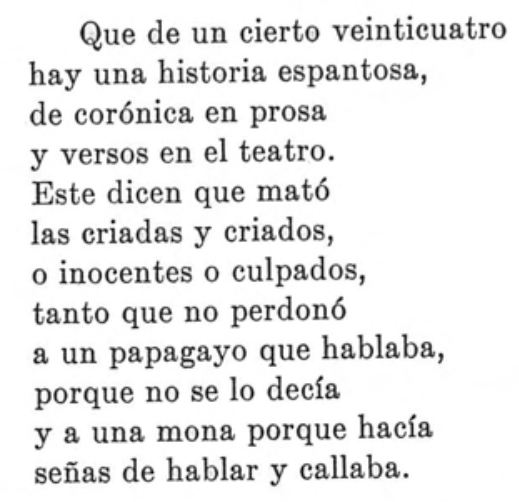

En resumen, hasta que no se establezca el texto original de Los comendadores de Córdoba, el que se supone escrito antes de 1593, no podremos formarnos idea completa del desarrollo que el gracioso había alcanzado para esa época. Y hasta que no sepamos las alteraciones que sufrió dicho texto no podremos juzgar el personaje de Galindo anteponiéndolo a Tristán.

\footnotetext{
${ }^{78}$ Montesinos, op. cit., 473, nota 3.

79 Jörder, op. cit., 276-77.

${ }^{80}$ Millé y Giménez, op. cit., 45.

${ }^{81}$ H. II, 462 a. $\quad$ Para la fecha, efr. Jörder, op. cit., 55.
} 


\section{EL FAVOR AGRADECIDO}

Esta comedia, cuyo primer acto está escrito y firmado de puño y letra de Lope, con fecha 19 de diciembre de 1593, nos ofrece el ejemplo más acabado de las figuras cómicas que preceden al Tristán de La francesilla. La mayoría de los críticos mantienen que Pinelo es un gracioso. Place caracteriza esta actitud: "Lope de Vega presents a bona fide gracioso type in his El favor agradecido, and with the following characteristics: he makes witty and humorously nonsensical speeches, parodies his master's love affair, and manifests chronic hunger and cowardice. Both this gracioso and that of Los comendadores de Córdoba appear so to speak, out of a clear sky: in the plays previously mentioned there is no evidence of experimentation pointing toward the gracioso formula, no gradual evolution, no putting together of a dash of bobo, a spoonful of lacayo, and a tincture of picaro. Lope may have happened on a copy of the Himenea . . . (de Torres Naharro) and may have said to himself, thrilled to the marrow: 'Eureka!' forthwith putting pen to paper to write Los comendadores de Córdoba with a gracioso; but such a coincidence must be questioned if more plausible possibilities can be alleged." 82

Indudablemente que debemos aceptar esta posibilidad cum grano salis, dada la disposición del dramaturgo y del público para quien escribía. Es dudabilísimo que si Lope descubrió la vena cómica del gracioso en 1593 lo abandonara durante dos años hasta llegar a La francesilla.

No hay duda que Pinelo tiene mucho de gracioso, pero hay algo en él que lo diferencia de la larga familia que lo había de suceder. No hallamos en él ni la burla ni la alabanza de su linaje, ni mucho menos la cómica parodia de las acciones y amores de su amo, aunque sí es cierto que al final de la pieza se casa con un apóstata princesa mora. Como todo buen gracioso, Pinelo es el consejero de su amo, pero sus consejos no son los que acostumbra dar el gracioso. El eterno consejo del gracioso para males de amor es el que hubiera dado Lope mismo: otros amores; Pinelo receta la caza y el ejercicio. El carácter aventurero de Pinelo, que se viste de moro con su amo; se introduce bajo falsa representación en la corte de un rey de Argel, huye arriesgando la vida en galeras turcas, desempeñando el papel de embajador en el tráfago del campamento, y otras tantas aventuras que pasamos por alto, dan a Pinelo cierto matiz de seriedad que no es propio del gracioso. Así no es de extrañar que Rennert le niegue

${ }^{82}$ Place, op. cit., 260-61. 
gracioso a esta comedia, o que Buchanan, admitiendo que Pinelo es un gracioso, diga: "There is nothing Spanish about him." Y, hablando luego de la comedia en general: "This play goes far to show that the New or Lopesque Comedia did not exist in 1593; certain elements were still lacking." ${ }^{83}$ Y la nueva comedia lopesca, tal cual la interpretamos hoy día, presupone al gracioso acabado.

Del análisis que hemos hecho de las comedias de Lope que se suponen o que sabemos fueron escritas antes de 1595 se desprenden las siguientes conclusiones:

1. Debido a errores cronológicos la crítica había creído hallar el gracioso en comedias anteriores a 1593.

2. Debido a errores de interpretación literaria se la había otorgado la denominación de gracioso a otros personajes más o menos cómicos que aparecen en dramas escritos en 1593 y 1594.

3. Estos años constituyen una época de ensayo con respecto al elemento cómico, en la cual se concretan gradualmente los rasgos característicos del gracioso.

4. De estos precursores del gracioso, el Galindo de Los comendadores de Córdoba y el Pinelo de El favor agradecido son ejemplos casi perfectos; pero en el caso del primero falta material para confirmar la fecha de composición de la comedia, o por lo menos, no sabemos hasta qué punto Lope retocó la comedia, y por lo tanto, al gracioso, al publicarla; y en el caso del segundo, la crítica moderna varía en su interpretación de la figura del donaire.

5. Por consiguiente, es preciso admitir ahora que es posible que Lope tenga razón cuando dice que el gracioso salió por primera vez a las tablas españolas en La francesilla.

6. Pero, aun admitiendo que Los comendadores de Córdoba y $\mathrm{El}$ favor agradecido amenacen este argumento, podemos retrasar la entrada del gracioso sólo dos años, y para los efectos de la cronología podemos aceptar como posteriores a 1593 las comedias que tengan graciosos.

7. Las siguientes comedias mencionadas en la primera lista de El peregrino que tienen graciosos pertenecen, pues, a la década 1593-1603.

El enemigo engañado; El primer rey de Castilla; La serrana de Tormes; El caballero del milagro; El mármol de Felisardo; El vaquero de Moraña; La varona castellana; Los torneos de Aragon; La divina vencedora; El mesón de la cortes; Los donaires de Matico; El lacayo fingido.

83 Buchanan, Modern Language Notes, 1909, 199. 
Las siguientes comedias, cuyo terminus ad quem ha sido establecido por la crítica, se remontan a más tardar al 1593:

$\begin{array}{ll}1593-1600 & \text { Los Guzmanes de Toral } \\ 1593-1602 & \text { El remedio en la desdicha } \\ 1593-1598 & \text { El galán escarmentado } \\ 1593-1598 & \text { La serrana de la Vera } \\ 1593-1598 & \text { La traición bien acertada }\end{array}$

Hemos excluído de esta lista las comedias en que a la presencia del gracioso y a la inclusión en la primera edición de $E l$ peregrino se opone el peso de una versificación de época más tardía, lo cual indica que fueron refundidas. Hemos excluído tambien comedias de dudosa atribución a Lope. ${ }^{84}$ Nos hemos circunscrito meramente a las diez y siete comedias que toda la evidencia indica que fueron escritas en la década comprendida entre 1593-1603.

J. H. ArJona

\section{Connecticut State College}

${ }^{84}$ Cfr. por ejemplo: El primer Fajardo, Antonio Roca, El nacimiento de Cristo, La lealtad en el agravio, El engaño en la verdad, etc. 